\title{
The First Ten Minutes
}

Rob Graham, R.R.T./N.R.C.P.

I dedicate this column to the late Dr. Andrew (Andy) Shennan, the founder of the perinatal program at Women's College Hospital (now at Sunnybrook Health Sciences Centre). To my teacher, my mentor and the man I owe my career as it is to, thank you. You have earned your place where there are no hospitals and no NICUs, where all the babies do is laugh and giggle and sleep.

"We are accustomed to referring to the first hour of a baby's life as "the golden hour." If the first hour is golden, then the first ten minutes of that hour are platinum."

We are accustomed to referring to the first hour of a baby's life as "the golden hour." If the first hour is golden, then the first ten minutes of that hour are platinum.

It is well known that the lungs are most prone to damage during recruitment. This is doubly so with the extremely premature infant since their lungs are so fragile and prone to airway and alveolar duct damage. Standard practices may not be appropriate with these infants; bag/mask ventilation pressures are typically higher than estimated even when delivered by an experienced, seasoned provider. Various factors affect delivered pressures. The leak around the face mask can be considerable(1), and endexpiratory pressures (PEEP) can be very difficult to provide consistently. In my experience, more recent flow inflating bags with an adjustable leak dial to provide PEEP are prone to either under or over providing PEEP. This is especially true when used with an endotracheal tube (ETT). A T-piece resuscitation device may provide more consistent peak inspiratory pressure (PIP)(2). It has been my experience that stable PEEP is also difficult to maintain with these devices.

With the advent of $\mathrm{CO}_{2}$ detectors, there is no excuse for assessing ETT placement by bagging with a high enough pressure to auscultate air entry. Manual bag ventilation should be avoided with an ETT in situ at all costs. Between flow graphics and adjustable manual inspiration pressures available on modern ventilators, this practice is technically archaic. It has long been suspected that the first few manual breaths given to a premature baby to assess ETT placement set that baby up for chronic lung disease (CLD) later.

I reported last month (The lowa Way) on practice by Dr. Klein and the outcomes of extremely premature infants in his NICU. In addition to using HFJV with this patient population, he aims to provide HFJV within 10 minutes of birth. This makes sense. Minimizing bag/mask ventilation and providing HFJV as quickly as possible may mitigate lung injury so commonly produced during initial resuscitation. The inflammatory response initiated at this point in conjunction with inflammatory factors associated with preterm birth may lead to CLD later. (4) We, I think, can all relate to the infant who cruises along splendidly on minimal ventilator settings and oxygen only to have everything escalate after about a week. This is the harvest of what has all too often been sown during those first 10 minutes.

"We, I think, can all relate to the infant who cruises along splendidly on minimal ventilator settings and oxygen only to have everything escalate after about a week. This is the harvest of what has all too often been sown during those first 10 minutes."

Proper nasal ETT placement can be estimated at six-plus double the weight in $\mathrm{kg}$. Oral tubes may be estimated at six plus the weight in $\mathrm{kg}$, although this formula is less precise than the one for nasal ETT placement. Newer formulae for oral ETT placement have been suggested as more reliable, such as four plus the birth weight in kg plus 0.05 times gestational age in weeks, but this requires further validation. (3) It is a commonplace in the unit in which I practice to administer surfactant when required based on these formulae and without radiographic verification of ETT placement.

Anecdotally, chronic lung disease (CLD) rates dropped in the unit where I practice when we connected babies to the ventilator immediately after intubation. The manual breath button was used in concert with flow graphics to assess ETT placement. With the addition of a $\mathrm{CO}_{2}$ detector, this method is almost foolproof and reduces the potentially damaging effects of manual ventilation with

NEONATOLOGY TODAY is interested in publishing manuscripts from Neonatologists, Fellows, NNPs and those involved in caring for neonates on case studies, research results, hospital news, meeting announcements, and other pertinent topics.

Please submit your manuscript to: LomaLindaPublishingCompany@gmail.com 
a resuscitation bag. Also, the ventilator provides stable, accurate PEEP and peak inspiratory pressures (PIP), unlike the manual resuscitation bag. Personal practice is to use HFO/VG at $10 \mathrm{~Hz}$ with VG set at $2 \mathrm{~mL} / \mathrm{kg}$ and manual inspiration set to $20 \mathrm{cmH}_{2} \mathrm{O}$ pressure as initial settings. Pressing the manual breath button until a blip appears on the flow graphic indicates flow, and colour change on the $\mathrm{CO}_{2}$ detector indicates that flow is into the baby's lungs. While flow sensors are not used when providing high-frequency jet ventilation (HFJV), the $\mathrm{CO}_{2}$ detector still confirms ETT placement.

\section{"While flow sensors are not used when providing high-frequency jet ventilation (HFJV), the $\mathrm{CO}_{2}$ detector still confirms ETT placement."}

While on the topic of targeted volumes, it is important to note that in order to be lung-protective, the lung must be recruited. A dialedin target of $4 \mathrm{~mL} / \mathrm{kg}$ is actually $8 \mathrm{~mL} / \mathrm{kg}$ if the lung is only half recruited, provides a false sense of security and little to no lung protection.

The cost of CLD is well known, not just while in hospital but postdischarge. Home oxygen, ventilation, and tracheostomy come with considerable costs. Whether born by a national health program or private insurance, this is money that could be much better spent. Here an ounce of prevention is indeed worth a pound of cure. Using $\mathrm{CO}_{2}$ detectors does involve the cost of the detector, about $\$ 10$. This is a small price to pay if the usage thereof decreases the rate of CLD. Of course, using the ventilator as quickly as possible has no associated costs above normal management whatsoever, and the potential savings are hardly insignificant. Newer ventilators also provide more stable CPAP when used in non-invasive mode.

\section{"The use of rapid sequence induction (RSI) has been shown to decrease cerebral blood flow changes with the associated risk of brain bleeds."}

The use of rapid sequence induction (RSI) has been shown to decrease cerebral blood flow changes with the associated risk of brain bleeds. It also increases the chance of successful intubation regardless of the skill of the clinician. (5) Wherever possible, RSI should be used during intubation, including during initial resuscitation(6). The skill and speed of the clinician charged with inserting umbilical lines can directly impact the timing of RSI, and it may not be possible to provide RSI in an emergent situation. A "flat" infant clearly requires immediate action. It is common practice to insert umbilical venous and arterial lines at the same time. It is typically much easier and quicker to place an umbilical venous line than an umbilical arterial line. Perhaps the umbilical venous line should be inserted first, and the arterial line placed after RSI intubation. The added time to split line placement into two procedures is short as re-prepping takes little time, and the person placing lines could, staff permitting, step back and remain sterile.

Early administration of surfactant may mitigate lung injury by reducing the inflammatory response. (7) This comes with its own problem: the rapid changes in compliance associated with surfactant administration. This change in compliance results in larger tidal volumes being delivered at a given pressure, regardless of the mode of ventilation used. This can result in very rapid changes in $\mathrm{PaCO}_{2}$ with resulting changes in cerebral vasculature tone and increases stretch injury to the lungs. While targeted volumes reduce the risk of stretch injury and hyperventilation post-surfactant, blood gas values must be carefully monitored during the first hour. Here, point of care testing is invaluable as turnaround time for laboratory-processed blood gases can be agonizingly long. A great deal can change in 30 minutes! I firmly believe that point of care testing for blood gas values (or a blood gas machine and personnel trained in its usage) should be available and mandatory in the resuscitation room. Unfortunately, this is not always the case.

\section{"Beyond the associated pulmonary sequelae, there are neurological consequences associated with the "golden hour." We know the premature infant is particularly susceptible to noxious stimuli, including pain, noise, and touch."}

Beyond the associated pulmonary sequelae, there are neurological consequences associated with the "golden hour." We know the premature infant is particularly susceptible to noxious stimuli, including pain, noise, and touch.

Most resuscitation rooms are much too loud. The noise monitor where I practice is all too often displaying red, indicating too much noise. The more people in the room, the louder it gets. Anyone not directly involved in resuscitation should, quite frankly, not be in the room, save a designated person for documentation. Necessary personnel should communicate as quietly as is practically possible. Light in the resuscitation room can be very high. Efforts should be made to shield the infant's eyes from strong light sources.

We strive for minimal handling during the first 72 hours of a micropremature infant's life. Given this, it makes sense to begin those 72 hours immediately. For instance, Infant length and head circumference do not need to be measured immediately although many clinicians seem compelled to do so. While not investigated, polyethylene occlusive wraps may reduce initial handling while reducing hypothermia. (8)

When it comes to the first hour, and especially the first 10 minutes, we should all be practicing with a Midas touch!

\section{Clarification}

In last month's column ("The lowa Way") the BPD/CLD rate in the sub-24 week gestation group was reported as $11 \%$. This is using the Jensen 'et al. definition of BPD, staged into 3 stratas(9), not 
the Shennan definition of BPD as requirement of supplemental oxygen at 36 weeks postmenstrual age. (10) By this definition the grade III BPD rate in the sub-24 week gestation group was approximately $11 \%$ in the $2016-2019$ inborn cohort who received HFJV as first intention. Survival at 22 weeks is reported at $58 \%$ and $74 \%$ at 23 weeks gestation, relatively high for this cohort. Survival rates of $25-50 \%$ have been reported elsewhere in the 22 week strata (11) and $50 \%$ in the 23 week strata. (12)

I thank Dr. Klein for this clarification and apologize for any misinterpretation that may have arisen.

\section{References:}

1. http://website60s.com/upload/files/16b.pdf

2. https://www.ncbi.n/m.nih.gov/pmc/articles/PMC4399249/

$3 \quad$ https://www.pediatr-neonatol.com/article/S18759572(18)30241-9/fulltext

4. $\quad$ https://link.springer.com/article/10.1007/s00281-020-00803-2

5. https://www.nature.com/articles/jp201432.pdf?origin $=p p u b$

6. https://www.cps.ca/en/documents/position/endotracheal-intubation-newborn

$7 \quad$ https://www.atsjournals.org/doi/full/10.1164/rccm.200912$18180 \mathrm{C}$

8. https://pubmed.ncbi.n/m.nih.gov/10228287/

9. $\quad$ https://www.ncbi.n/m.nih.gov/pmc/articles/PMC6775872/

10. https://www.atsjournals.org/doi/pdf/10.1513/ AnnalsATS.201504-2180C

11 https://pediatrics.aappublications.org/content/144/3/ $\underline{\text { e20190113 }}$

12. https://healthydebate.ca/2019/06/topic/babies-born23-weeks-preterm

Disclosures: The author receives compensation from Bunnell Inc for teaching and training users of the LifePulse HFJV in Canada. He is not involved in sales or marketing of the device nor does he receive more than per diem compensation. Also, while the author practices within Sunnybrook H.S.C. this paper should not be construed as Sunnybrook policy per se. This article contains elements considered "off label" as well as maneuvers, which may sometimes be very effective but come with inherent risks. As with any therapy, the riskbenefit ratio must be carefully considered before they are initiated.

.NT

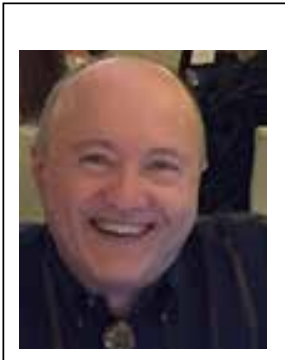

Corresponding Author

Rob Graham, R.R.T./N.R.C.P.

Advanced Practice Neonatal RRT

Sunnybrook Health Science Centre

43 Wellesley St. East

Toronto, ON

Canada M4Y $1 \mathrm{H1}$

Email: Rob Graham <rcgnrcp57@yahoo.ca>

Telephone: 416-967-8500
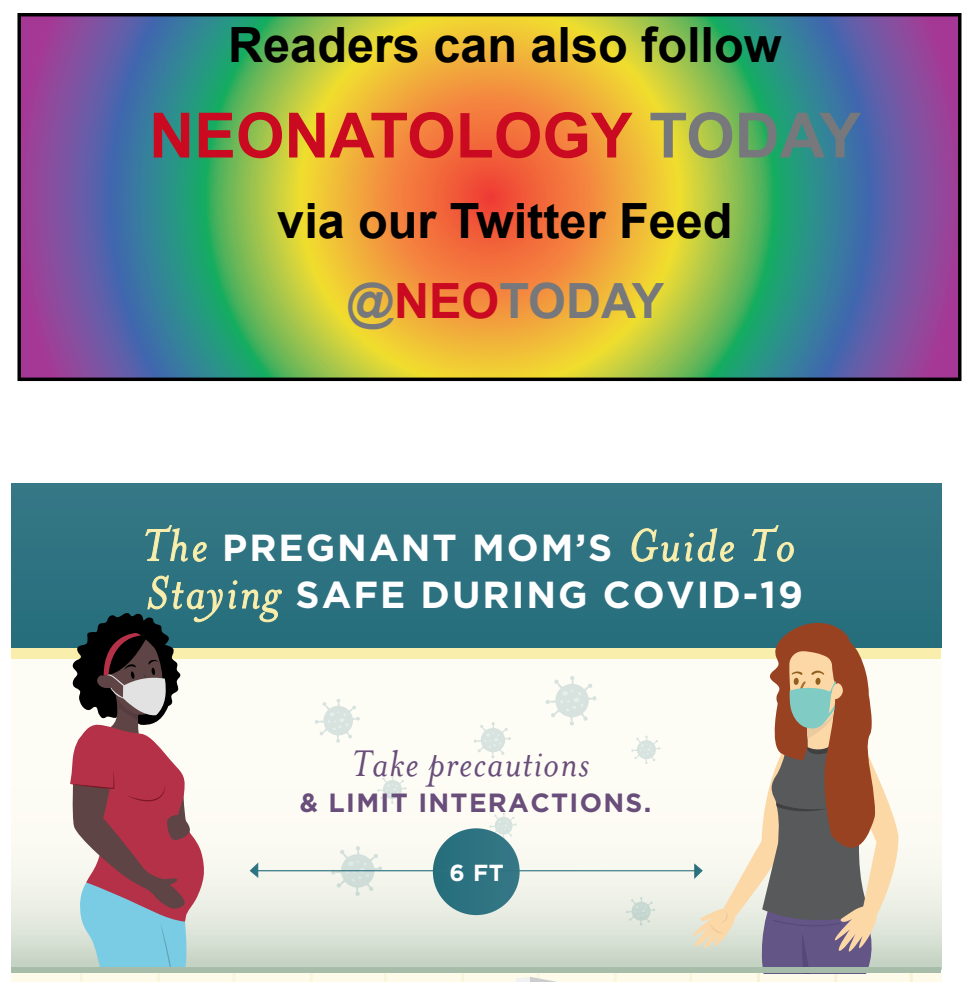

Maintain at least

A 30-DAY SUPPLY

OF YOUR MEDICATIONS.
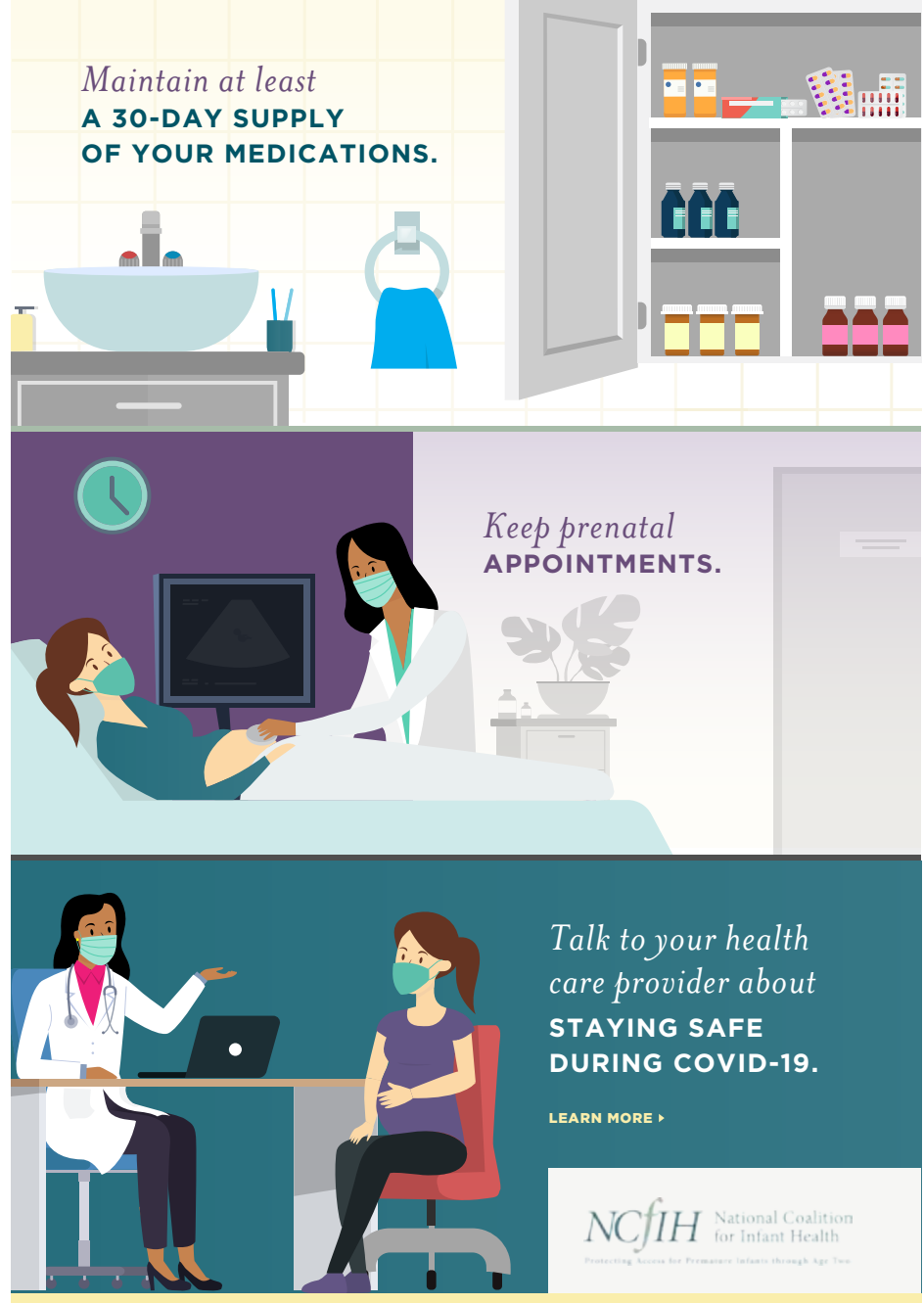